\title{
THE CP-MATRIX COMPLETION PROBLEM
}

\author{
ANWA ZHOU AND JINYAN FAN
}

\begin{abstract}
A symmetric matrix $C$ is completely positive (CP) if there exists an entrywise nonnegative matrix $B$ such that $C=B B^{T}$. The CP-completion problem is to study whether we can assign values to the missing entries of a partial matrix (i.e., a matrix having unknown entries) such that the completed matrix is completely positive. We propose a semidefinite algorithm for solving general CP-completion problems, and study its properties. When all the diagonal entries are given, the algorithm can give a certificate if a partial matrix is not $\mathrm{CP}$-completable, and it almost always gives a CP-completion if it is CP-completable. When diagonal entries are partially given, similar properties hold. Computational experiments are also presented to show how CP-completion problems can be solved.
\end{abstract}

\section{INTRODUCTION}

A matrix is partial if some of its entries are missing. The matrix completion problem is to study whether we can assign values to the missing entries of a partial matrix such that the completed matrix satisfies certain properties, e.g., it is positive semidefinite or an Euclidean distance matrix. This problem has wide applications, as shown in [3, 14, 28, 32, 36]. We refer to Laurent's survey [31] and the references therein. Interesting applications include the Netflix problem 29, 37, global positioning [13, multi-task learning [1|24], etc. The motivation of this paper is to study whether or not a partial matrix can be completed to a matrix that is completely positive.

A real $n \times n$ symmetric matrix $C$ is completely positive (CP) if there exist nonnegative vectors $u_{1}, \cdots, u_{m}$ in $\mathbb{R}^{n}$ such that

$$
C=u_{1} u_{1}^{T}+\cdots+u_{m} u_{m}^{T}
$$

where $m$ is called the length of the decomposition (1.1). The smallest $m$ in the above is called the CP-rank of $C$. If $C$ is complete positive, we call (1.1) a CPdecomposition of $C$. Clearly, a matrix $C$ is completely positive if and only if $C=B B^{T}$ for an entrywise nonnegative matrix $B$. Hence, a CP-matrix is not only positive semidefinite but also nonnegative entrywise.

CP-matrices have wide applications in mixed binary quadratic programming [11, approximating stability numbers [16], max clique problems [43,47, single quadratic constraint problems [4], standard quadratic optimization problems [7, and general quadratic programming [45. Some NP-hard problems can be formulated as linear optimization problems over the cone of CP-matrices (cf. [22, 27, 34]). We refer

2000 Mathematics Subject Classification. Primary 15A23, 15A48, 15A83, 90C22.

$K e y$ words and phrases. completely positive matrices, matrix completion, $E$-matrices, $\mathcal{E}$ truncated $\Delta$-moment problem, semidefinite program.

The second author is partially supported by NSFC 11171217 . 
to [5, 6, 8, 10, 12, 17, 19, for the work in this field. These important applications motivate us to study the so-called CP-completion problem. Let

$$
E=\left\{\left(i_{k}, j_{k}\right) \mid 1 \leq i_{k} \leq j_{k} \leq n, k=1, \cdots, l\right\} .
$$

be an index set of pairs. A partial symmetric matrix $A$ is called an $E$-matrix if its entries $A_{i j}$ are given for all $(i, j) \in E$, while $A_{i j}$ are missing for $(i, j) \notin E$. The CP-completion problem is to study whether we can assign values to the missing entries of an $E$-matrix such that the completed matrix is completely positive. If such an assignment exists, we say that the $E$-matrix is CP-completable; otherwise, we say that it is not CP-completable (or non-CP-completable).

A symmetric matrix can be identified by a vector that consists of its upper triangular entries. Similarly, an $E$-matrix $A$ can be identified as a vector

$$
\mathbf{a} \in \mathbb{R}^{E},
$$

such that $\mathbf{a}_{i j}=A_{i j}$ for all $(i, j) \in E$. (The symbol $\mathbb{R}^{E}$ stands for the space of all real vectors indexed by pairs in $E$.) For a matrix $F$, we denote by $\left.F\right|_{E}$ the vector in $\mathbb{R}^{E}$ whose $(i, j)$-entry is $F_{i j}$, for all $(i, j) \in E$. Clearly, an $E$-matrix $A$ is $\mathrm{CP}$-completable if and only if there exists a CP-matrix $C$ such that $\mathbf{a}=\left.C\right|_{E}$.

For example, consider the $E$-matrix $A$ given as ( [5, Example 2.23]):

$$
\left[\begin{array}{llll}
2 & 3 & 0 & * \\
3 & 6 & 3 & 0 \\
0 & 3 & 6 & 3 \\
* & 0 & 3 & 2
\end{array}\right]
$$

where $*$ means that the entry there is missing, throughout the paper. The index set $E$ is

$$
\{(1,1),(1,2),(1,3),(2,2),(2,3),(2,4),(3,3),(3,4),(4,4)\},
$$

and the identifying vector a of $A$ is

$$
(2,3,0,6,3,0,6,3,2) \text {. }
$$

If we assign the missing entry $A_{14}$ a value, say, $t$, the determinant of $A$ is $-27(t+1)$. So, $A$ can not be positive semidefinite for any $t>-1$. This implies that the $E$ matrix $A$ is not CP-completable.

For another example, consider the $E$-matrix $A$ given as:

$$
\left[\begin{array}{lll}
1 & 1 & 1 \\
1 & 1 & 1 \\
1 & 1 & *
\end{array}\right]
$$

The index set $E$ is $\{(1,1),(1,2),(1,3),(2,2),(2,3)\}$ and the identifying vector $\mathbf{a}$ is $(1,1,1,1,1)$. Since

$$
\left[\begin{array}{lll}
1 & 1 & 1 \\
1 & 1 & 1 \\
1 & 1 & 1
\end{array}\right]=\left(\begin{array}{l}
1 \\
1 \\
1
\end{array}\right)\left(\begin{array}{l}
1 \\
1 \\
1
\end{array}\right)^{T}
$$

is completely positive, we know that this $E$-matrix is CP-completable.

Note that if a diagonal entry of a CP-matrix is zero, then all the entries in its row or column are zeros. Without loss of generality, we can assume that all the given entries of an $E$-matrix are nonnegative and all its given diagonal entries are strictly positive. Otherwise, it can be reduced to a smaller $E^{\prime}$-matrix with some index set $E^{\prime}$. 
An $E$-matrix $A$ is called a partial CP-matrix if every principal submatrix of $A$, whose entry indices are all from $E$, is completely positive. When all the diagonal entries are given, the specification graph of an $E$-matrix is defined as the graph whose vertex set is $\{1,2, \ldots, n\}$ and whose edge set is $\{(i, j) \in E: i \neq j\}$. A symmetric partial matrix is called a matrix realization of a graph $G$ if its specification graph is $G$. It is shown in [5,20] that a partial CP-matrix realization of a connected graph $G$ is CP-completable if and only if $G$ is a block-clique graph. Under some conditions, a partial CP-matrix, whose specification graph $G$ contains cycles, is CP-completable if and only if all the blocks of $G$ are cliques or cycles [21. These results assume that all the diagonal entries are given and the specification graphs satisfy certain combinatorial properties.

In this paper, we study general CP-completion problems in a unified framework. If an $E$-matrix is not CP-completable, how can we get a certificate for this? If it is $\mathrm{CP}$-completable, how can we get a CP-completion and a CP-decomposition for the completed matrix? To the best knowledge of the authors, there exists few work on solving general CP-completion problems.

The paper is organized as follows. In Section 2, we give a semidefinite algorithm for solving general CP-completion problems, after an introduction of truncated moment problems. Its basic properties are also studied. In Section 3, we study properties of CP-completion problems when some diagonal entries are missing. Computational results are given in Section 4. Finally, we conclude the paper in Section 5 with some applications and discussions about future work.

\section{A SEMIDEFinite ALGORITHM FOR CP-COMPLETION}

Recently, Nie 38 proposed a semidefinite algorithm for solving $\mathcal{A}$-truncated $K$-moment problems ( $\mathcal{A}$-TKMPs), which are generalizations of classical truncated $K$-moment problems (cf. 24]). In this section, we show how to formulate CPcompletion problems in the framework of $\mathcal{A}$-TKMP, and then propose a semidefinite algorithm to solve them.

2.1. CP-completion as $\mathcal{E}$-T $\Delta \mathbf{M P}$. First, we characterize when an $E$-matrix is CP-completable. Let

$$
\Delta=\left\{x \in \mathbb{R}^{n}: x_{1}+\cdots+x_{n}-1=0, x_{1} \geq 0, \ldots, x_{n} \geq 0\right\}
$$

be the standard simplex in $\mathbb{R}^{n}$. For convenience, denote the polynomials:

$$
h(x):=x_{1}+\cdots+x_{n}-1, g_{1}(x):=x_{1}, \ldots, g_{n}(x):=x_{n} .
$$

Let $A$ be an $E$-matrix with the identifying vector $\mathbf{a} \in \mathbb{R}^{E}$. We have seen that $A$ is CP-completable if and only if $\mathbf{a}=\left.C\right|_{E}$ for some CP-matrix $C$. Note that every nonnegative vector is a multiple of a vector in the simplex $\Delta$. So, in view of (1.1), $A$ is CP-completable if and only if there exist vectors $v_{1}, \cdots, v_{m} \in \Delta$ and $\rho_{1}, \cdots, \rho_{m}>0$ such that

$$
C=\rho_{1} v_{1} v_{1}^{T}+\cdots+\rho_{m} v_{m} v_{m}^{T} \quad \text { and } \quad \mathbf{a}=\left.C\right|_{E} .
$$

Let $\mathbb{N}$ be the set of nonnegative integers. For $\alpha=\left(\alpha_{1}, \ldots, \alpha_{n}\right) \in \mathbb{N}^{n}$, denote $|\alpha|:=\alpha_{1}+\cdots+\alpha_{n}$. Let $\mathbb{N}_{d}^{n}:=\left\{\alpha \in \mathbb{N}^{n}:|\alpha| \leq n\right\}$. Denote

$$
\mathcal{E}:=\left\{\alpha \in \mathbb{N}^{n}: \alpha=e_{i}+e_{j},(i, j) \in E\right\},
$$

where $\mathbf{e}_{i}$ is the $i$-th unit vector. For instance, when $n=3$ and $E=\{(1,2),(2,2),(2,3)\}$, then $\mathcal{E}=\{(1,1,0),(0,2,0),(0,1,1)\}$. The degree $\operatorname{deg}(\mathcal{E}):=\max \{|\alpha|: \alpha \in \mathcal{E}\}$ is 
two for all $E$. Since there is a one-to-one correspondence between $\mathcal{E}$ and $E$, we can also index the identifying vectors $\mathbf{a} \in \mathbb{R}^{E}$ of $E$-matrices as

$$
\mathbf{a}=\left(\mathbf{a}_{\alpha}\right)_{\alpha \in \mathcal{E}} \in \mathbb{R}^{\mathcal{E}}, \quad \mathbf{a}_{\alpha}=\mathbf{a}_{i j} \quad \text { if } \quad \alpha=e_{i}+e_{j} .
$$

$\left(\mathbb{R}^{\mathcal{E}}\right.$ denotes the space of real vectors indexed by elements in $\mathcal{E}$.) We call such a an $\mathcal{E}$-truncated moment sequence ( $\mathcal{E}$-tms) (cf. [38]).

The $\mathcal{E}$-truncated $\Delta$-moment problem $(\mathcal{E}$-T $\Delta \mathrm{MP})$ studies whether or not a given $\mathcal{E}$-tms a admits a $\Delta$-measure $\mu$, i.e., a nonnegative Borel measure $\mu$ supported in $\Delta$ such that

$$
\mathbf{a}_{\alpha}=\int_{\Delta} x^{\alpha} d \mu \quad \forall \alpha \in \mathcal{E}
$$

where $x^{\alpha}:=x_{1}^{\alpha_{1}} \cdots x_{n}^{\alpha_{n}}$. A measure $\mu$ satisfying the above is called a $\Delta$-representing measure for a. A measure is called finitely atomic if its support is a finite set, and is called $m$-atomic if its support consists of at most $m$ distinct points. We refer to 38 for representing measures of truncated moment sequences.

Hence, by (2.2), an $E$-matrix $A$, with the identifying vector $\mathbf{a} \in \mathbb{R}^{\mathcal{E}}$, is $\mathrm{CP}$ completable if and only if a admits an $m$-atomic $\Delta$-measure, i.e.,

$$
\mathbf{a}=\rho_{1}\left[v_{1}\right]_{\mathcal{E}}+\cdots+\rho_{m}\left[v_{m}\right]_{\mathcal{E}},
$$

with each $v_{i} \in \Delta$ and $\rho_{i}>0$. In the above, we denote

$$
[v]_{\mathcal{E}}:=\left(v^{\alpha}\right)_{\alpha \in \mathcal{E}} .
$$

In other words, $\mathrm{CP}$-completion problems are equivalent to $\mathcal{E}$-T $\Delta \mathrm{MPs}$ with $\mathcal{E}$ and $\Delta$ given by (2.3) and (2.1) respectively.

2.2. A semidefinite algorithm. We present a semidefinite algorithm for solving CP-completion problems, by formulating them as $\mathcal{E}$-T $\Delta$ MPs. To describe it, we need to introduce localizing matrices. Denote

$$
\mathbb{R}[x]_{\mathcal{E}}:=\operatorname{span}\left\{x^{\alpha}: \alpha \in \mathcal{E}\right\} .
$$

We say that $\mathbb{R}[x]_{\mathcal{E}}$ is $\Delta$-full if there exists a polynomial $p \in \mathbb{R}[x]_{\mathcal{E}}$ such that $\left.p\right|_{\Delta}>0$ (cf. [42]). Let $\mathbb{R}[x]_{d}:=\operatorname{span}\left\{x^{\alpha}: \alpha \in \mathbb{N}_{d}^{n}\right\}$. An $\mathcal{E}$-tms $y \in \mathbb{R}^{\mathcal{E}}$ defines an $\mathcal{E}$-Riesz function $\mathscr{L}_{y}$ acting on $\mathbb{R}[x]_{\mathcal{E}}$ as

$$
\mathscr{L}_{y}\left(\sum_{\alpha \in \mathcal{E}} p_{\alpha} x^{\alpha}\right):=\sum_{\alpha \in \mathcal{E}} p_{\alpha} y_{\alpha} .
$$

For $z \in \mathbb{R}^{\mathbb{N}_{2 k}^{n}}$ and $q \in \mathbb{R}[x]_{2 k}$, the $k$-th localizing matrix of $q$ generated by $z$ is the symmetric matrix $L_{q}^{(k)}(z)$ satisfying

$$
\mathscr{L}_{z}\left(q p^{2}\right)=\operatorname{vec}(p)^{T}\left(L_{q}^{(k)}(z)\right) \operatorname{vec}(p) \quad \forall p \in \mathbb{R}[x]_{k-\lceil\operatorname{deg}(q) / 2\rceil} .
$$

In the above, $\operatorname{vec}(p)$ denotes the coefficient vector of $p$ in the graded lexicographical ordering, and $\lceil t\rceil$ denotes the smallest integer that is not smaller than $t$. In particular, when $q=1, L_{1}^{(k)}(z)$ is called a $k$-th order moment matrix and denoted as $M_{k}(z)$. We refer to [38,40] for more details about localizing and moment matrices.

Let $g_{0}(x):=1$ and $g_{n+1}(x):=1-\|x\|_{2}^{2}$. Since $\Delta \subseteq B(0,1):=\left\{x \in \mathbb{R}^{n}:\|x\|_{2}^{2} \leq\right.$ $1\}$, we can also describe $\Delta$ equivalently as

$$
\Delta=\left\{x \in \mathbb{R}^{n}: h(x)=0, g(x) \geq 0\right\},
$$


where $g(x):=\left(g_{0}(x), g_{1}(x), \ldots, g_{n}(x), g_{n+1}(x)\right)$. For instance, when $n=2$ and $k=2$, the second order localizing matrices of the above polynomials are:

$$
\begin{aligned}
& L_{x_{1}+x_{2}-1}^{(2)}(z)=\left[\begin{array}{lll}
z_{(1,0)}+z_{(0,1)}-z_{(0,0)} & z_{(2,0)}+z_{(1,1)}-z_{(1,0)} & z_{(1,1)}+z_{(0,2)}-z_{(0,1)} \\
z_{(2,0)}+z_{(1,1)}-z_{(1,0)} & z_{(3,0)}+z_{(2,1)}-z_{(2,0)} & z_{(2,1)}+z_{(1,2)}-z_{(1,1)} \\
z_{(1,1)}+z_{(0,2)}-z_{(0,1)} & z_{(2,1)}+z_{(1,2)}-z_{(1,1)} & z_{(1,2)}+z_{(0,3)}-z_{(0,2)}
\end{array}\right], \\
& M_{2}(z):=L_{1}^{(2)}(z)=\left[\begin{array}{llllll}
z_{(0,0)} & z_{(1,0)} & z_{(0,1)} & z_{(2,0)} & z_{(1,1)} & z_{(0,2)} \\
z_{(1,0)} & z_{(2,0)} & z_{(1,1)} & z_{(3,0)} & z_{(2,1)} & z_{(1,2)} \\
z_{(0,1)} & z_{(1,1)} & z_{(0,2)} & z_{(2,1)} & z_{(1,2)} & z_{(0,3)} \\
z_{(2,0)} & z_{(3,0)} & z_{(2,1)} & z_{(4,0)} & z_{(3,1)} & z_{(2,2)} \\
z_{(1,1)} & z_{(2,1)} & z_{(1,2)} & z_{(3,1)} & z_{(2,2)} & z_{(1,3)} \\
z_{(0,2)} & z_{(1,2)} & z_{(0,3)} & z_{(2,2)} & z_{(1,3)} & z_{(0,4)}
\end{array}\right], \\
& L_{x_{1}}^{(2)}(z)=\left[\begin{array}{lll}
z_{(1,0)} & z_{(2,0)} & z_{(1,1)} \\
z_{(2,0)} & z_{(3,0)} & z_{(2,1)} \\
z_{(1,1)} & z_{(2,1)} & z_{(1,2)}
\end{array}\right], \quad L_{x_{2}}^{(2)}(z)=\left[\begin{array}{lll}
z_{(0,1)} & z_{(1,1)} & z_{(0,2)} \\
z_{(1,1)} & z_{(2,1)} & z_{(1,2)} \\
z_{(0,2)} & z_{(1,2)} & z_{(0,3)}
\end{array}\right], \\
& L_{1-x_{1}^{2}-x_{2}^{2}}^{(2)}(z)=\left[\begin{array}{ccc}
z_{(0,0)}-z_{(2,0)}-z_{(0,2)} & z_{(1,0)}-z_{(3,0)}-z_{(1,2)} & z_{(0,1)}-z_{(2,1)}-z_{(0,3)} \\
z_{(1,0)}-z_{(3,0)}-z_{(1,2)} & z_{(2,0)}-z_{(4,0)}-z_{(2,2)} & z_{(1,1)}-z_{(3,1)}-z_{(1,3)} \\
z_{(0,1)}-z_{(2,1)}-z_{(0,3)} & z_{(1,1)}-z_{(3,1)}-z_{(1,3)} & z_{(0,2)}-z_{(2,2)}-z_{(0,4)}
\end{array}\right] \text {. }
\end{aligned}
$$

As shown in [38, a necessary condition for $z \in \mathbb{R}^{\mathbb{N}_{2 k}^{n}}$ to admit a $\Delta$-measure is

$$
L_{h}^{(k)}(z)=0, \quad \text { and } \quad L_{g_{j}}^{(k)}(z) \succeq 0, \quad j=0,1, \ldots, n+1 .
$$

(In the above, $X \succeq 0$ means that $X$ is positive semidefinite.) If, in addition to (2.8), $z$ satisfies the rank condition

$$
\operatorname{rank} M_{k-1}(z)=\operatorname{rank} M_{k}(z),
$$

then $z$ admits a unique $\Delta$-measure, which is $\operatorname{rank} M_{k}(z)$-atomic (cf. Curto and Fialkow [15). We say that $z$ is flat if (2.8) and (2.9) are both satisfied.

Given two tms' $y \in \mathbb{R}^{\mathbb{N}_{d}^{n}}$ and $z \in \mathbb{R}^{\mathbb{N}_{e}^{n}}$, we say $z$ is an extension of $y$, if $d \leq e$ and $y_{\alpha}=z_{\alpha}$ for all $\alpha \in \mathbb{N}_{d}^{n}$. We denote by $\left.z\right|_{\mathcal{E}}$ the subvector of $z$, whose entries are indexed by $\alpha \in \mathcal{E}$. For convenience, we denote by $\left.z\right|_{d}$ the subvector $\left.z\right|_{\mathbb{N}_{d}^{n}}$. If $z$ is flat and extends $y$, we say $z$ is a flat extension of $y$. Note that an $\mathcal{E}$-tms $\mathbf{a} \in \mathbb{R}^{\mathcal{E}}$ admits a $\Delta$-measure if and only if it is extendable to a flat tms $z \in \mathbb{R}^{\mathbb{N}_{2 k}^{n}}$ for some $k$ (cf. 38). By (2.5), determining whether an $E$-matrix $A$ is CP-completable or not is equivalent to investigating whether a has a flat extension or not.

Let $d>2$ be an even integer. Choose a polynomial $R \in \mathbb{R}[x]_{d}$ and write it as

$$
R(x)=\sum_{\alpha \in \mathbb{N}_{d}^{n}} R_{\alpha} x^{\alpha} .
$$

Consider the linear optimization problem

$$
\begin{array}{ll}
\min _{z} & \sum_{\alpha \in \mathbb{N}_{d}^{n}} R_{\alpha} z_{\alpha} \\
\text { s.t. } & \left.z\right|_{\mathcal{E}}=\mathbf{a}, z \in \Upsilon_{d}(\Delta),
\end{array}
$$

where $\Upsilon_{d}(\Delta)$ is the set of all tms' $z \in \mathbb{R}^{\mathbb{N}_{d}^{n}}$ admitting $\Delta$-measures. Note that $\Delta$ is a compact set. It is shown in [38] that if $\mathbb{R}[x]_{\mathcal{E}}$ is $\Delta$-full, then the feasible set of (2.10) is compact convex and (2.10) has a minimizer for all $R$. If $\mathbb{R}[x]_{\mathcal{E}}$ is not $\Delta$-full, we need to choose $R$ which is positive definite on $\Delta$, to guarantee that (2.10) has a minimizer. Therefore, to get a minimizer of (2.10), it is enough to solve (2.10) for a generic positive definite $R$, no matter whether $\mathbb{R}[x]_{\mathcal{E}}$ is $\Delta$-full or not. For this reason, we choose $R \in \Sigma_{n, d}$, where $\Sigma_{n, d}$ is the set of all sum of squares polynomials 
in $n$ variables with degree $d$. Since $\Upsilon_{d}(\Delta)$ is typically quite difficult to describe, we relax it by the cone

$$
\Gamma_{k}(h, g):=\left\{z \in \mathbb{R}^{\mathbb{N}_{2 k}^{n}} \mid L_{h}^{(k)}(z)=0, L_{g_{j}}^{(k)}(z) \succeq 0, j=0,1, \ldots, n+1\right\},
$$

with $k \geq d / 2$ an integer. The $k$-th order semidefinite relaxation of (2.10) is

$$
(S D R)_{k}:\left\{\begin{array}{cl}
\min _{z} & \sum_{\alpha \in \mathbb{N}_{d}^{n}} R_{\alpha} z_{\alpha} \\
\text { s.t. } & \left.z\right|_{\mathcal{E}}=\mathbf{a}, z \in \Gamma_{k}(h, g) .
\end{array}\right.
$$

Based on solving the hierarchy of (2.12), our semidefinite algorithm for solving CP-completion problems is as follows.

Algorithm 2.1. A semidefinite algorithm for solving CP-completion problems.

Step 0: Choose a generic $R \in \Sigma_{n, d}$, and let $k:=d / 2$.

Step 1: Solve (2.12). If (2.12) is infeasible, then a doesn't admit a $\Delta$-measure, i.e., $A$ is not CP-completable, and stop. Otherwise, compute a minimizer $z^{*, k}$. Let $t:=1$.

Step 2: Let $w:=\left.z^{*, k}\right|_{2 t}$. If the rank condition (2.9) is not satisfied, go to Step 4 .

Step 3: Compute the finitely atomic measure $\mu$ admitted by $w$ :

$$
\mu=\lambda_{1} \delta\left(u_{1}\right)+\cdots+\lambda_{m} \delta\left(u_{m}\right),
$$

where $m=\operatorname{rank} M_{t}(w), u_{i} \in \Delta, \lambda_{i}>0$, and $\delta\left(u_{i}\right)$ is the Dirac measure supported on the point $u_{i}(i=1, \cdots, m)$. Stop.

Step 4: If $t<k$, set $t:=t+1$ and go to Step 2; otherwise, set $k:=k+1$ and go to Step 1.

Remark 2.2. Algorithm 2.1 is a specialization of Algorithm 4.2 in 38 to CPcompletion. Denote $[x]_{d}:=\left(x^{\alpha}\right)_{\alpha \in \mathbb{N}_{d}^{n}}$. We choose $R=[x]_{d / 2}^{T} J^{T} J[x]_{d / 2}$ in (2.12), where $J$ is a random square matrix obeying Gaussian distribution. We check the rank condition (2.9) numerically with the help of singular value decompositions [23]. The rank of a matrix is evaluated as the number of its singular values that are greater than or equal to $10^{-6}$. We use the method in [25] to get a $m$-atomic $\Delta$ measure for $w$.

2.3. Some properties of Algorithm 2.1. We first show some basic properties of Algorithm 2.1 which are from [38, Section 5].

Theorem 2.3. Algorithm 2.1 has the following properties:

1) If (2.12) is infeasible for some $k$, then a admits no $\Delta$-measures and the corresponding E-matrix $A$ is not $C P$-completable.

2) If the E-matrix $A$ is not CP-completable and $\mathbb{R}[x]_{\mathcal{E}}$ is $\Delta$-full, then (2.12) is infeasible for all $k$ big enough.

3) If the E-matrix $A$ is CP-completable, then for almost all generated $R$, we can asymptotically get a flat extension of a by solving the hierarchy of (2.12). This gives a $C P$-completion of $A$.

Remark 2.4. Under some general conditions, which is almost sufficient and necessary, we can get a flat extension of a by solving the hierarchy of (2.12), within finitely many step (cf. [38). This always happens in our numerical experiments. So, if an $E$-matrix $A$ with the identifying vector $\mathbf{a} \in \mathbb{R}^{\mathcal{E}}$ is CP-completable, then we can asymptotically get a flat extension of a for almost all $R \in \Sigma_{n, d}$ by Algorithm 
2.1. Moreover, it can often be obtained within finitely many steps. After getting a flat extension of $\mathbf{a}$, we can get a $m$-atomic $\Delta$-measure for $\mathbf{a}$, which then produces a CP-completion of $A$, as well as a CP-decomposition.

When $\mathbb{R}[x]_{\mathcal{E}}$ is $\Delta$-full, Algorithm 2.1 can give a certificate for the non-CPcompletability. However, if it is not $\Delta$-full and $A$ is not CP-completable, it is not clear whether there exists a $k$ such that (2.12) is infeasible. This is an open question, to the best knowledge of the authors. We now characterize when $\mathbb{R}[x]_{\mathcal{E}}$ is $\Delta$-full.

Proposition 2.5. Suppose $E=\left\{\left(i_{k}, j_{k}\right) \mid 1 \leq i_{k} \leq j_{k} \leq n, k=1, \cdots, l\right\}$. Then, $\mathbb{R}[x]_{\mathcal{E}}$ is $\Delta$-full if and only if $\{(i, i): 1 \leq i \leq n\} \subseteq E$.

Proof. We first prove the sufficient condition. If $\{(i, i), 1 \leq i \leq n\} \subseteq E$, then $\{(2,0, \cdots, 0),(0,2, \cdots, 0), \cdots,(0,0, \cdots, 2)\} \subseteq \mathcal{E}$, so we have $x_{i}^{2} \in \mathbb{R}[x]_{\mathcal{E}}$ for all $1 \leq i \leq n$. Hence for any $x \in \Delta$, there exists a polynomial $p(x)=\sum_{i=1}^{n} x_{i}^{2} \in \mathbb{R}[x]_{\mathcal{E}}$ such that $p(x)>0$. Thus $\mathbb{R}[x]_{\mathcal{E}}$ is $\Delta$-full.

We prove the necessary condition by contradiction. Suppose there exists some $i_{0} \in\{1, \cdots, n\}$ such that $\left(i_{0}, i_{0}\right) \notin E$. For any polynomial $p(x) \in \mathbb{R}[x]_{\mathcal{E}}, p(x)$ is a linear combination of all the monomials of degree 2 except $x_{i_{0}}^{2}$. Let $c=$ $\left(0, \cdots, 0,1_{i_{0}}, 0, \cdots, 0\right)^{T} \in \Delta$ be a constant vector, then $p(c)=0$ holds for all $p(x) \in$ $\mathbb{R}[x]_{\mathcal{E}}$. Hence, there does not exit any polynomial $p(x) \in \mathbb{R}[x]_{\mathcal{E}}$ such that $\left.p(x)\right|_{\Delta}>$ 0 . This contradicts the fact that $\mathbb{R}[x]_{\mathcal{E}}$ is $\Delta$-full. The proof is completed.

Remark 2.6. Proposition 2.5 shows that $\mathbb{R}[x]_{\mathcal{E}}$ is $\Delta$-full if and only if all the diagonal entries are given. In such case, Algorithm 2.1 can determine whether an $E$-matrix $A$ can be completed to a CP-matrix or not. If $A$ is CP-completable, Algorithm 2.1 can give a CP-completion with a CP-decomposition. If $A$ is not CP-completable, then it can give a certificate, i.e., (2.12) is infeasible for some $k$.

\section{CP-COMPLETION With MisSing DiagOnal ENTRIES}

When all the diagonal entries are given, which is equivalent to that $\mathbb{R}[x]_{\mathcal{E}}$ is $\Delta$-full, the properties of Algorithm 2.1 are summarized in Theorem 2.3 and Remark 2.6. In this section, we study properties of CP-completion problems when some diagonal entries are missing.

3.1. All diagonal entries are missing. Consider $E$-matrices with all the diagonal entries missing, i.e., $(i, i) \notin E$ for all $i$. In such case, CP-completion is relatively simple, as shown below.

Proposition 3.1. Let $A$ be an E-matrix whose entries are all nonnegative. If all the diagonal entries of $A$ are missing, then $A$ has a CP-completion.

Proof. The matrix

$$
C=\sum_{(i, j) \in E} A_{i j}\left(\mathbf{e}_{i}+\mathbf{e}_{j}\right)\left(\mathbf{e}_{i}+\mathbf{e}_{j}\right)^{T}
$$

is clearly completely positive, because each $A_{i j} \geq 0$. It is easy to check that $C$ is a CP-completion of $A$.

Remark 3.2. By Proposition 3.1 every nonnegative $E$-matrix is CP completable, when all diagonal entries are missing. In such case, Algorithm 2.1 typically gives a CP-decomposition whose length is much smaller than the length in the proof of 
Proposition 3.1, which is the cardinality of $E$. This is an advantage of Algorithm 2.1 .

3.2. Diagonal entries are partially missing. We consider $E$-matrices whose diagonal entries are not all missing, i.e., the set $E$ contains at least one but not all of $(1,1), \ldots,(n, n)$. Suppose the diagonal entry indices in $E$ are $\left(i_{1}, i_{1}\right), \ldots,\left(i_{r}, i_{r}\right)$. Let

$$
\hat{E}=\left\{(i, j) \in E: i, j \in\left\{i_{1}, \ldots, i_{r}\right\}\right\} .
$$

Let $A$ be an $E$-matrix. An $\hat{E}$-matrix $P$ is called the maximum principal submatrix of $A$ if $P_{i j}=A_{i j}$ for all $(i, j) \in \hat{E}$. If $P$ is CP-completable, we say that $A$ is partially CP-completable. Clearly, if $A$ is CP-completable, then $P$ is also CP-completable. This immediately leads to the following proposition.

Proposition 3.3. If the maximum principal submatrix of an E-matrix $A$ is not $C P$-completable, then $A$ is not CP-completable.

Remark 3.4. The converse of Proposition 3.3 is not necessarily true. For example, consider the $E$-matrix $A$ given as

$$
\left[\begin{array}{lll}
1 & 1 & 2 \\
1 & 1 & 3 \\
2 & 3 & *
\end{array}\right] .
$$

The determinant of $A$ is always -1 , no matter what the $(3,3)$-entry is. So, it can not be positive semidefinite, and hence $A$ is not $\mathrm{CP}$-completable. However, its maximum principal submatrix $P$ is completely positive, so $A$ is partially CPcompletable.

Though the $E$-matrix $A$ in (3.2) is not CP-completable, we can show that there exists a sequence $\left\{A_{k}\right\}$ of CP-completable $E$-matrices such that their identifying vectors converge to the one of $A$.

Theorem 3.5. Suppose the maximum principal submatrix of an E-matrix A, with the identifying vector $\mathbf{a} \in \mathbb{R}^{\mathcal{E}}$, is CP-completable. Then there exists a sequence of $C P$-completable E-matrices $\left\{A_{k}\right\}$, whose identifying vectors converge to a.

Proof. If all the diagonal entries are given, the theorem is clearly true.

First, we assume exactly one diagonal entry is missing. Without loss of generality, we assume $A$ is given in the following form

$$
\left[\begin{array}{cccc} 
& & & A_{1, n} \\
& A^{\prime} & & \vdots \\
& & & A_{n-1, n} \\
A_{n, 1} & \cdots & A_{n, n-1} & *
\end{array}\right],
$$

where $A^{\prime}$ is the maximum principal submatrix of $A$. If some of the entries $A_{i, n}, A_{n, i}$ $(i=1, \ldots, n-1)$ are missing, we assign the constant value 1 to them. The matrix $A^{\prime}$ is CP-completable, by assumption, and all its diagonal entries are given. Consider the following sequence of $E$-matrices:

$$
\left[\begin{array}{cccc} 
& & & A_{1, n} \\
& & & \vdots \\
& A^{\prime}+\varepsilon_{k} I_{n-1} & & A_{n-1, n} \\
A_{n, 1} & \cdots & A_{n, n-1} & *
\end{array}\right], \quad k=1,2, \cdots,
$$


where $I_{n-1}$ is the identity matrix of order $n-1$ and $0<\varepsilon_{k} \rightarrow 0$ as $k \rightarrow \infty$. Let $\overline{A^{\prime}}$ be a CP-completion of $A^{\prime}$, and

$$
A_{k}=\left[\begin{array}{ll}
\overline{A^{\prime}} & \mathbf{0} \\
\mathbf{0}^{T} & 1
\end{array}\right]+\sum_{1 \leq i \leq n-1}\left(\sqrt{\varepsilon_{k}} \mathbf{e}_{i}+{\sqrt{\varepsilon_{k}}}^{-1} A_{i, n} \mathbf{e}_{n}\right)\left(\sqrt{\varepsilon_{k}} \mathbf{e}_{i}+{\sqrt{\varepsilon_{k}}}^{-1} A_{i, n} \mathbf{e}_{n}\right)^{T},
$$

where $\mathbf{0}$ is the zero vector. Clearly, $A_{k}$ is a CP-completion of the matrix in (3.4), and the sequence $\left\{\left.A_{k}\right|_{E}\right\}$ converges to $\mathbf{a}$ as $k \rightarrow+\infty$.

Second, when two or more diagonal entries are missing, the proof is same as in the above. We omit it here for cleanness.

Remark 3.6. Theorem 3.5 implies that the set of all $C P$-completable $E$-matrices is not closed, if some diagonal entries are missing.

When an $E$-matrix has only one given diagonal entry, there is a nice property of CP-completion.

Proposition 3.7. Let $A$ be a nonnegative E-matrix. If only one diagonal entry of $A$ is given and it is positive, then $A$ is CP-completable.

Proof. Without loss of generality, we assume the first diagonal is given and positive. Let $\tilde{n}$ be the number of the given entries in the first row. So, $1 \leq \tilde{n} \leq n$. If $\tilde{n}=1$, let

$$
C=A_{11} \mathbf{e}_{1} \mathbf{e}_{1}^{T}+\sum_{2 \leq i<j \leq n,(i, j) \in E} A_{i j}\left(\mathbf{e}_{i}+\mathbf{e}_{j}\right)\left(\mathbf{e}_{i}+\mathbf{e}_{j}\right)^{T} .
$$

Then $C$ is a CP-completion of $A$. Otherwise, if $\tilde{n}>1$, let

$$
\begin{aligned}
C= & \sum_{1=i<j \leq n,(1, j) \in E}\left(\sqrt{\frac{A_{11}}{\tilde{n}-1}} \mathbf{e}_{1}+\sqrt{\frac{\tilde{n}-1}{A_{11}}} A_{1 j} \mathbf{e}_{j}\right)\left(\sqrt{\frac{A_{11}}{\tilde{n}-1}} \mathbf{e}_{1}+\sqrt{\frac{\tilde{n}-1}{A_{11}}} A_{1 j} \mathbf{e}_{j}\right)^{T} \\
& +\sum_{2 \leq i<j \leq n,(i, j) \in E} A_{i j}\left(\mathbf{e}_{i}+\mathbf{e}_{j}\right)\left(\mathbf{e}_{i}+\mathbf{e}_{j}\right)^{T} .
\end{aligned}
$$

It can be easily checked that $C$ is a CP-completion of $A$.

Remark 3.8. 1) If an $E$-matrix is not partially CP-completable, a certificate (i.e., the relaxation (2.12) is infeasible) can be obtained by applying Algorithm 2.1 to its maximum principle submatrix. 2) If an $E$-matrix is partially CP-completable, then Algorithm 2.1 can give a CP-completion, up to an arbitrarily tiny positive perturbation (applied to given diagonal entries).

\section{NumericAl EXPERIMENTS}

In this section, we present numerical experiments for solving CP-completion problems by using Algorithm 2.1. We use software GloptiPoly 3 [26] and SeDuMi [46] to solve semidefinite relaxations in (2.12). We choose $d=4$ and $k=2$ in Step 0 of Algorithm 2.1.

Example 4.1. Consider the $E$-matrix $A$ given as (cf. [5, Exercise 2.57]):

$$
\left[\begin{array}{llll}
b & 3 & 0 & * \\
3 & 6 & 3 & 0 \\
0 & 3 & 6 & 3 \\
* & 0 & 3 & b
\end{array}\right],
$$


where $b \geq 0$ is a parameter. For a symmetric nonnegative matrix of order $n \leq 4$, it is completely positive if and only if it is positive semidefinite (cf. [33]), i.e., all its principal minors are nonnegative. Let $c=A_{14}$, the missing value. Then $A$ is completely positive if and only if

$$
b \geq 0, c \geq 0,2 b-3 \geq 0, b-2 \geq 0,2 b^{2}-3 b-2 c^{2} \geq 0,(b-2)^{2}-(c+1)^{2} \geq 0 .
$$

The above is satisfiable if and only if $b \geq 3$, i.e., $A$ is CP-completable if and only if $b \geq 3$. When $b=3, A$ is CP-completable only for $c=0$.

We choose $b=3$ and apply Algorithm 2.1. It terminates at Step 3 with $k=3$, and gives the $\mathrm{CP}$-completion

$$
A=\left[\begin{array}{cccc}
3 & 3 & 0 & 0.0000 \\
3 & 6 & 3 & 0 \\
0 & 3 & 6 & 3 \\
0.0000 & 0 & 3 & 3
\end{array}\right]=\sum_{i=1}^{3} \rho_{i} u_{i} u_{i}^{T},
$$

where $u_{i}$ and $\rho_{i}$ are given in Table 1

\begin{tabular}{|c|c|c|}
\hline$i$ & $u_{i}$ & $\rho_{i}$ \\
\hline 1 & $(0.5000,0.5000,0.0000,0.0000)^{T}$ & 12.0000 \\
\hline 2 & $(0.0000,0.0000,0.5000,0.5000)^{T}$ & 12.0000 \\
\hline 3 & $(0.0000,0.5000,0.5000,0.0000)^{T}$ & 12.0000 \\
\hline
\end{tabular}

TABLE 1. The points $u_{i}$ and weights $\rho_{i}$ in Example 4.1.

Example 4.2. Consider the $E$-matrix $A$ given as:

$$
\left[\begin{array}{lllll}
* & 4 & 1 & 2 & 2 \\
4 & * & 0 & 1 & 3 \\
1 & 0 & * & 1 & 2 \\
2 & 1 & 1 & * & 1 \\
2 & 3 & 2 & 1 & *
\end{array}\right]
$$

All its diagonal entries are missing. By Proposition 3.1, we know $A$ is CP-completable. We apply Algorithm 2.1, It terminates at Step 3 with $k=3$, and gives the CPcompletion:

$$
\left[\begin{array}{ccccc}
5.8127 & 4 & 1 & 2 & 2 \\
4 & 4.6697 & 0 & 1 & 3 \\
1 & 0 & 2.2682 & 1 & 2 \\
2 & 1 & 1 & 0.9087 & 1 \\
2 & 3 & 2 & 1 & 4.7740
\end{array}\right]=\sum_{i=1}^{3} \rho_{i} u_{i} u_{i}^{T},
$$

where $u_{i}$ and $\rho_{i}$ are shown in Table 2. The length of the CP-decomposition is 3,

\begin{tabular}{|c|c|c|}
\hline$i$ & $u_{i}$ & $\rho_{i}$ \\
\hline 1 & $(0.1595,0.0000,0.3619,0.1595,0.3191)^{T}$ & 17.3224 \\
\hline 2 & $(0.1122,0.4258,0.0000,0.0000,0.4620)^{T}$ & 13.9667 \\
\hline 3 & $(0.4957,0.3179,0.0000,0.1488,0.0376)^{T}$ & 21.1443 \\
\hline
\end{tabular}

TABLE 2. The points $u_{i}$ and weights $\rho_{i}$ in Example 4.2 . 
which is much shorter than 9 given by (3.1). This shows an advantage of Algorithm 2.1

Example 4.3. Consider the $E$-matrix $A$ given as

$$
\left[\begin{array}{ccccc}
6.1232 & 4.1232 & 1.1233 & 2.1231 & 2.3321 \\
4.1232 & * & 0 & 1.0987 & 3.2873 \\
1.1233 & 0 & 3.2318 & 1.2332 & 2.1232 \\
2.1231 & 1.0987 & 1.2332 & * & 1.1232 \\
2.3321 & 3.2873 & 2.1232 & 1.1232 & *
\end{array}\right]
$$

By Proposition [2.5, the space $\mathbb{R}[x]_{\mathcal{E}}$ is not $\Delta$-full. We apply Algorithm [2.1] It terminates at Step 3 with $k=4$, and gives the CP-completion:

$$
\left[\begin{array}{ccccc}
6.1232 & 4.1232 & 1.1233 & 2.1231 & 2.3321 \\
4.1232 & 5.5494 & 0 & 1.0987 & 3.2873 \\
1.1233 & 0 & 3.2318 & 1.2332 & 2.1232 \\
2.1231 & 1.0987 & 1.2332 & 1.0430 & 1.1232 \\
2.3321 & 3.2873 & 2.1232 & 1.1232 & 3.6641
\end{array}\right]=\sum_{i=1}^{4} \rho_{i} u_{i} u_{i}^{T},
$$

where $u_{i}$ and $\rho_{i}$ are shown in Table 3 . This also shows a nice property of Algorithm

\begin{tabular}{|c|c|c|}
\hline$i$ & $u_{i}$ & $\rho_{i}$ \\
\hline 1 & $(0.3499,0.3637,0.0000,0.0807,0.2057)^{T}$ & 32.4007 \\
\hline 2 & $(0.0000,0.5555,0.0000,0.0650,0.3795)^{T}$ & 4.0941 \\
\hline 3 & $(0.4805,0.0000,0.2503,0.2692,0.0000)^{T}$ & 9.3406 \\
\hline 4 & $(0.0000,0.0000,0.4925,0.1124,0.3951)^{T}$ & 10.9106 \\
\hline
\end{tabular}

TABLE 3. The points $u_{i}$ and weights $\rho_{i}$ in Example 4.3 .

2.1 if it exists, a CP-completion can be found, even if $\mathbb{R}[x]_{\mathcal{E}}$ is not $\Delta$-full.

Example 4.4. Consider the $E$-matrix $A$ given as:

$$
\left[\begin{array}{llllll}
* & 7 & 1 & 3 & 9 & * \\
7 & * & 5 & 8 & 5 & 3 \\
1 & 5 & * & 2 & 2 & * \\
3 & 8 & 2 & 3 & 1 & 4 \\
9 & 5 & 2 & 1 & * & 1 \\
* & 3 & * & 4 & 1 & *
\end{array}\right]
$$

Only one diagonal entry is given. By Proposition 3.7 (4.4) is CP-completable. We apply Algorithm 2.1. It terminates at Step 3 with $k=5$, and gives the CPcompletion

$$
\left[\begin{array}{cccccc}
11.3758 & 7 & 1 & 3 & 9 & 6.1225 \\
7 & 32.5203 & 5 & 8 & 5 & 3 \\
1 & 5 & 4.2013 & 2 & 2 & 2.5314 \\
3 & 8 & 2 & 3 & 1 & 4 \\
9 & 5 & 2 & 1 & 11.1114 & 1 \\
6.1225 & 3 & 2.5314 & 4 & 1 & 10.8581
\end{array}\right]=\sum_{i=1}^{9} \rho_{i} u_{i} u_{i}^{T},
$$

where $u_{i}$ and $\rho_{i}$ are listed in Table 4. 


\begin{tabular}{|c|c|c|}
\hline$i$ & $u_{i}$ & $\rho_{i}$ \\
\hline 1 & $(0.0000,0.0458,0.5132,0.0224,0.4187,0.0000)^{T}$ & 1.9950 \\
\hline 2 & $(0.0000,0.4881,0.2702,0.1191,0.1225,0.0000)^{T}$ & 5.6983 \\
\hline 3 & $(0.0434,0.5268,0.1914,0.1203,0.1183,0.0000)^{T}$ & 41.6816 \\
\hline 4 & $(0.0000,0.0000,0.3857,0.1581,0.0000,0.4562)^{T}$ & 11.2576 \\
\hline 5 & $(0.1508,0.5697,0.0000,0.1599,0.0000,0.1197)^{T}$ & 44.0288 \\
\hline 6 & $(0.1929,0.5399,0.0000,0.1050,0.1622,0.0000)^{T}$ & 17.9222 \\
\hline 7 & $(0.2977,0.0000,0.0324,0.1558,0.0000,0.5141)^{T}$ & 29.2892 \\
\hline 8 & $(0.4287,0.0842,0.0000,0.0000,0.4871,0.0000)^{T}$ & 11.0745 \\
\hline 9 & $(0.4121,0.0000,0.0306,0.0000,0.4875,0.0697)^{T}$ & 29.4268 \\
\hline
\end{tabular}

TABLE 4 . The points $u_{i}$ and weights $\rho_{i}$ in Example 4.4 .

Example 4.5. Consider the $E$-matrix $A$ given as (cf. [5, Example 1.35]):

$$
\left[\begin{array}{lllll}
1 & 1 & * & * & 0 \\
1 & 1 & 1 & * & * \\
* & 1 & 1 & 1 & * \\
* & * & 1 & 1 & 1 \\
0 & * & * & 1 & 1
\end{array}\right] .
$$

It is shown in [5] that (4.5) is not CP-completable. We apply Algorithm 2.1 to verify this fact. It terminates at Step 1 with $k=3$ as (2.12) is infeasible, which confirms that (4.5) is not CP-completable.

Example 4.6. Consider the $E$-matrix $A$ given as:

$$
\left[\begin{array}{lllll}
1 & 1 & 2 & * & 4 \\
1 & 1 & 3 & * & 3 \\
2 & 3 & 3 & 3 & * \\
* & * & 3 & 2 & * \\
4 & 3 & * & * & *
\end{array}\right] .
$$

By Proposition 2.5, the set $\mathbb{R}[x]_{\mathcal{E}}$ is not $\Delta$-full. We apply Algorithm 2.1 to solve this CP-completion problem. It terminates at Step 1 with $k=1$, because (2.12) is infeasible. This shows that the $E$-matrix $A$ is not CP-completable. By this example, we can see that Algorithm 2.1 might get a certificate for non-CP-completability, even if $\mathbb{R}[x]_{\mathcal{E}}$ is not $\Delta$-full.

Example 4.7. Consider the $E$-matrix $A$ given as in Remark 3.4.

$$
\left[\begin{array}{lll}
1 & 1 & 2 \\
1 & 1 & 3 \\
2 & 3 & *
\end{array}\right]
$$

We have already seen that $A$ is not CP-completable. We apply positive perturbations to $A$ as follows:

$$
\left[\begin{array}{ccc}
1+10^{-l} & 1 & 2 \\
1 & 1+10^{-l} & 3 \\
2 & 3 & *
\end{array}\right], \quad l=1,2, \cdots
$$


By the proof of Theorem 3.5. we know that (4.8) is CP-completable for all $l$. For $l=1,2,3$, Algorithm 2.1 produces the following CP-completions:

$$
\begin{aligned}
A_{1} & =\left[\begin{array}{ccc}
1.1 & 1 & 2 \\
1 & 1.1 & 3 \\
2 & 3 & 10.9524
\end{array}\right]=\sum_{i=1}^{2} \lambda_{i} u_{i} u_{i}^{T}, \\
A_{2} & =\left[\begin{array}{ccc}
1.01 & 1 & 2 \\
1 & 1.01 & 3 \\
2 & 3 & 56.2189
\end{array}\right]=\sum_{i=1}^{2} \rho_{i} v_{i} v_{i}^{T},
\end{aligned}
$$

and

$$
A_{3}=\left[\begin{array}{ccc}
1.001 & 1 & 2 \\
1 & 1.001 & 3 \\
2 & 3 & 487.2967
\end{array}\right]=\sum_{i=1}^{4} \sigma_{i} \omega_{i} \omega_{i}^{T} .
$$

The points and their weights are shown in Tables 5 and 6 .

\begin{tabular}{|c|c|c|c|c|}
\hline$i$ & $u_{i}$ & $\lambda_{i}$ & $v_{i}$ & $\rho_{i}$ \\
\hline 1 & $(0.1254,0.1881,0.6866)^{T}$ & 23.2350 & $(0.0327,0.0490,0.9183)^{T}$ & 66.6636 \\
\hline 2 & $(0.6190,0.3810,0.0000)^{T}$ & 1.9174 & $(0.5124,0.4876,0.0000)^{T}$ & 3.5753 \\
\hline
\end{tabular}

TABLE 5. The points and weights for $A_{1}$ and $A_{2}$ in Example 4.7 .

\begin{tabular}{|c|c|c|}
\hline$i$ & $\omega_{i}$ & $\sigma_{i}$ \\
\hline 1 & $(0.5012,0.4987,0.0001)^{T}$ & 3.7527 \\
\hline 2 & $(0.0682,0.0696,0.8623)^{T}$ & 11.6502 \\
\hline 3 & $(0.0027,0.0048,0.9925)^{T}$ & 485.9000 \\
\hline 4 & $(0.4545,0.3039,0.2487)^{T}$ & 0.0023 \\
\hline
\end{tabular}

TABLE 6. The points and weights for $A_{3}$ in Example 4.7 .

When $l \geq 4$, the resulting semidefinite relaxations (2.12) are ill-conditioned, and the semidefinite programming solver SeDuMi has trouble to solve them accurately. This is because this $E$-matrix is not CP-completable, but it has an arbitrarily tiny perturbation that is $\mathrm{CP}$-completable.

\section{Conclusions and Discussions}

This paper proposes a semidefinite algorithm (i.e., Algorithm 2.1) for solving general CP-completion problems. When all the diagonal entries are given, Algorithm 2.1 can give a certificate for non-CP-completability. If a partial matrix is CP-completable, Algorithm 2.1 almost always gets a CP-completion, as well as a $\mathrm{CP}$-decomposition. When some diagonal entries are missing, if the maximum principal submatrix is not $\mathrm{CP}$-completable, then a certificate for non-CP-completability can be obtained; if it is $\mathrm{CP}$-completable, then Algorithm2.1 also almost always gives a CP-completion.

CP-completion has wide applications (cf. [5]). Here we show one in probability theory. Let $x$ be a random vector in $\mathbb{R}^{n}$. Suppose its expectation $\mathbb{E} x=b$ and partial entries of its covariance matrix are known, say, for an index set $E$ the entries

$$
X_{i j}=\mathbb{E}\left[\left(x_{i}-b_{i}\right)\left(x_{j}-b_{j}\right)\right]
$$


with $(i, j) \in E$ are known. We want to investigate for what values of $X_{i j}((i, j) \in E)$ the density function of $x$ is supported in the nonnegative orthant $\mathbb{R}_{+}^{n}$. This question is basic and natural, because many statistical quantities are positive in the world. Interestingly, this question can be formulated as a CP-completion problem. From the expression of $X_{i j}$, we can see that

$$
\mathbb{E}\left(x_{i} x_{j}\right)=X_{i j}+b_{i} b_{j} .
$$

Let $A$ be the $E$-matrix such that $A_{i j}=X_{i j}+b_{i} b_{j}$ for all $(i, j) \in E$. Whether the random vector $x$ has a density function supported in $\mathbb{R}_{+}^{n}$ or not is basically equivalent to whether the following partial matrix

$$
\left[\begin{array}{cc}
* & b^{T} \\
b & A
\end{array}\right]
$$

is CP-completable or not. In the above, $A$ is also a partial matrix; only the entries $A_{i j}$ with $(i, j) \in E$ are known.

CP-completion also has applications in nonconvex quadratic optimization. Let $E$ be an index set. A typical quadratic optimization problem is

$$
\left\{\begin{array}{cl}
\min & \sum_{(i, j) \in E} a_{i j} x_{i} x_{j} \\
\text { s.t. } & p(x)=1, x \geq 0
\end{array}\right.
$$

where $p(x)=\sum_{(i, j) \in E} p_{i j} x_{i} x_{j}$ is a given polynomial. To solve this nonconvex optimization problem globally, we need to characterize the cone

$$
\mathcal{C}_{E}=\left\{y \in \mathbb{R}^{E}: y=\left.C\right|_{E} \text { for some CP-matrix } C \in \mathbb{R}^{n \times n}\right\} .
$$

It can be shown that (5.1) is equivalent to the linear convex problem

$$
\left\{\begin{array}{cl}
\min & \sum_{(i, j) \in E} a_{i j} y_{i j} \\
\text { s.t. } & \sum_{(i, j) \in E} p_{i j} y_{i j}=1, y \in \mathcal{C}_{E} .
\end{array}\right.
$$

To design efficient numerical methods for solving (5.2), we need to check the memberships in $\mathcal{C}_{E}$. This is equivalent to solving $\mathrm{CP}$-completion problems.

In this paper, we mainly focus on determining whether a partial matrix is CPcompletable or not. However, we did not discuss the question of how to get a CP-completion whose CP-rank is minimum. This question is hard, and there exists few work about it, to the best knowledge of the authors. This is an interesting future work.

\section{REFERENCES}

[1] F. Abernethy, T. Evgeniou, J. P. Vert, Low-rank matrix factorization with attributes, Technical Report N24/06/MM. Paris, France: Ecole des Mines de Paris, 2006.

[2] F. Abernethy, T. Evgeniou, J. P. Vert, A new approach to collaborative filtering: operator estimation with spectral regularization, J. Mach. Learn. Res., 10 (2009), PP. 803-826.

[3] S. Al-Homidan, H. Wolkowicz, Approximate and exact completion problems for Euclidean distance matrices using semidefinite programming, Linear algebra and its applications, 406 (2005), PP. 109-141.

[4] A. Argyriou, T. Evgeniou, M. Pontil, Convex multi-task feature learning. Mach. Learn., 73 (2008), pp. 243-272.

[5] A. Berman and N. Shaked-Monderer, Completely Positive Matrices, World Scientific, 2003.

[6] A. Berman and D. Shasha, Completely positive house matrices, Linear Algebra and its Applications, 436 (2012), pp. 12-26. 
[7] I. M. Bomze, M. Dür, E. DeKlerk, C. Roos, A. J. Quist, and T. Terlaky, On copositive programming and standard quadratic optimization problems, J. Global Optim., 18 (2000), pp. 301-320.

[8] I. M. Bomze, Copositive optimization-recent developments and applications, Eur. J. Oper. Res. 216 (2012), pp. 509-520.

[9] I. M. Bomze, W. Schachinger, And G. Uchida, Think co(mpletely)positive! Matrix properties, examples and a clustered bibliography on copositive optimization, J Glob Optim, 52 (2012), pp. 423-445.

[10] S. Bundfuss, Copositive matrices, copositive programming, and applications, in Ph.D. Dissertation, TU Darmstadt, 2009. Online at http://www3.mathematik.tu-darmstadt.de/index.php?id=483

[11] S. BURER, On the copositive representation of binary and continuous nonconvex quadratic programs, Mathematical Programming, Ser. A, 120 (2009), pp. 479-495.

[12] S. Burer, Copositive programming, In: M. F. Anjos, J. B. Lasserre, (Eds.), Handbook on Semi-definite, Conic and Polynomial Optimization International Series in Operations Research \& Management Science, Springer, New York, 166 (2012), pp. 201-218.

[13] E. J. CAndès And Y. Plan, Matrix completion with noise, Proc. IEEE, 98 (2009), pp. 925936

[14] E. J. Candès And B. Recht, Exact Matrix Completion via Convex Optimization. Found Comput Math, 9 (2009), pp. 717-772.

[15] R. Curto And L. Fialkow, Truncated K-moment problems in several variables, Journal of Operator Theory, 54 (2005), pp. 189-226.

[16] E. De Klerk and D. V. Pasechnik, Approximation of the stability number of a graph via copositive programming, SIAM Journal on Optimization, 12 (2002), pp. 875-892.

[17] P. J. Dickinson, Geometry of the copositive and completely positive cones, J. Math. Anal. Appl., 380 (2011), pp. 377-395.

[18] P. J. Dickinson AND M. DüR, Linear-time complete positivity detection and decomposition of sparse matrices, SIAM Journal On Matrix Analysis and Applications, 33 (2012), pp. 701-720.

[19] P. J. Dickinson And L. GijBen, On the computational complexity of membership problems for the completely positive cone and its dual, Technical Report, Johann Bernoulli Institute for Mathematics and Computer Science, University of Groningen, The Netherlands, 2011.

[20] J. H. Drew And C. R. Johnson, The completely positive and doubly nonnegative completion problems, Linear and Multilinear Algebra, 44 (1998), pp. 85-92.

[21] J. H. Drew, C. R. Johnson, S. J. Kilner, And A. M. McKay, The cycle completable graphs for the completely positive and doubly nonnegative completion problems, Linear Algebra Appl., 313 (2000), pp. 141-154.

[22] M. Dür, Copositive Programming-a Survey, in M. Diehl, F. Glineur, E. Jarlebring, W. Michiels, Eds., Recent Advances in Optimization and its Applications in Engineering, Springer, 2010, pp. 3-20.

[23] G. H. Golub and C. F. Van Loan, Matrix computations, Third edition., The Johns Hopkins University Press, 1996.

[24] J. W. HeLton And J. Nie, A semidefinite approach for truncated K-moment problems, Foundations of Computational Mathematics, 12 (2012), pp. 851-881.

[25] D. Henrion and J. Lasserre, Detecting global optimality and extracting solutions in GloptiPoly, Positive polynomials in control, Lecture Notes in Control and Inform. Sci. Springer, Berlin, 312 (2005), pp. 293-310.

[26] D. Henrion, J. Lasserre, And J. LoefBerg, GloptiPoly 3: moments, optimization and semidefinite programming, Optimization Methods and Software, 24 (2009), pp. 761-779.

[27] J. B. Hiriart-Urruty and A. SeEger, A variational approach to copositive matrices, SiAM Rev., 52 (2010), pp. 593-629.

[28] H. X. Huang, Z. A. Liang, P. M. Pardalos, Some properties for the Euclidean distance matrix and positive semidefinite matrix completion problems, Journal of Global Optimization, 25 (2003), pp. 3-21.

[29] B. James and L. Stan, The netflix prize. Proceedings of KDD Cup and Workshop. (2007). Available at http://www.cs.uic.edu/Xliub/KDD-cup-2007/proceedings.html

[30] J. B. LASSERre, Global optimization with polynomials and the problem of moments, SIAM J. Optim., 11 (2001), pp. 796-817. 
[31] M. Laurent, Matrix completion problems, In The Encyclopedia of Optimization, Kluwer Academic, (2001), pp. 221-229.

[32] M. LAUREnt, Polynomial instances of the positive semidefinite and Euclidean distance matrix completion problems, SIAM Journal on Matrix Analysis and Applications, 22 (2001), pp. 874 894.

[33] J. E. Maxfield and H. Minc, On the equation $X^{T} X=A$. Proc. Edinburgh Math. Soc., 13 (1962), pp. 125-129.

[34] K. G. Murty And S. N. Kabadi, Some NP-complete problems in quadratic and nonlinear programming, Math. Programming, 39 (1987), pp. 117-129.

[35] S. Negahban AND M. J. Wainwright, Estimation of (near) low-rank matrices with noise and high-dimensional scaling. Ann. Statist. 39 (2011), 1069-1097.

[36] S. Negahban AND M. J. Wainwright, Restricted strong convexity and weighted matrix completion: Optimal bounds with noise. J. Mach. Learn. Res. 13 (2012), 1665-1697.

[37] Netflix, Netflix Prize. (2006). Available at http://www.netflixprize.com/

[38] J. NiE, The $\mathcal{A}$-truncated K-moment problem, arXiv:1210.6930 [math.FA].

[39] J. NIE, Discriminants and nonnegative polynomials, Journal of Symbolic Computation, 47 (2012), pp. 167-191.

[40] J. NiE, Certifying convergence of Lasserres hierarchy via flat truncation, Math. Program., Ser. A, (2012), to appear.

[41] J. NiE, Optimality conditions and finite convergence of Lasserres hierarchy, Mathematical Programming, (2013), DOI: 10.1007/s10107-013-0680-x.

[42] L. FiALKOW AND J. NiE, The truncated moment problem via homogenization and flat extensions, Journal of Functional Analysis, 263 (2012), pp. 1682-1700.

[43] P. M. Pardalos And G. P. Rodgers, A branch and bound algorithm for the maximum clique problem, Comp. Oper. Res., 19 (1992), pp. 363-375.

[44] J. C. PreIsig, Copositivity and the minimization of quadratic functions with nonnegativity and quadratic equality constraints, SIAM J. Control Optim., 34 (1996), pp. 1135-1150.

[45] A. J. Quist, E. De Klerk, C. Roos, and T. Terlaky, Copositive relaxation for general quadratic programming, Optimization Methods and Software, 9 (1998), pp. 185-208.

[46] J. F. STURM, SeDuMi 1.02: A MATLAB toolbox for optimization over symmetric cones, Optimization Methods and Software, 11 \& 12 (1999), pp. 625-653.

[47] J. ŽILINSKAs AND M. Dür, Depth-first simplicial partition for copositivity detection, with an application to MaxClique, Optimization Methods \& Software, 26 (2011), pp. 499-510.

Department of Mathematics, Shanghai Jiao Tong University, Shanghai 200240, P.R. CHINA

E-mail address: congcongyan@sjtu.edu.cn

Department of Mathematics, and MOE-LSC, Shanghai Jiao Tong University, ShangHAI 200240, P.R. CHINA

E-mail address: jyfan@sjtu.edu.cn 